\title{
The long-term effects of probiotics in the therapy of ulcerative colitis: A clinical study
}

\author{
Vincenzo Davide Palumbo ${ }^{\mathrm{a}, \mathrm{b}}$, Marcello Romeoc, Antonella Marino Gammazzaa, ${ }^{\mathrm{a}, \mathrm{c}}$ Francesco Carini ${ }^{\mathrm{c}, \mathrm{d}}$, Provvidenza Damiani ${ }^{\mathrm{d}}$, \\ Giuseppe Damianoc, Salvatore Buscemic, Attilio Ignazio Lo Montec, ${ }^{c, d}$, Alice Gerges-Geageac,e, Abdo Jurjuse, \\ Giovanni Tomasello ${ }^{\mathrm{a}, \mathrm{c}, \mathrm{d}}$
}

\begin{abstract}
Aim. Intestinal dysbiosis seems to be the leading cause of inflammatory bowel diseases, and probiotics seems to represent the proper support against their occurrence. Actually, probiotic blends and anti-inflammatory drugs represent a weapon against inflammatory bowel diseases. The present study evaluates the long-term (2 years) effects of combination therapy (mesalazine plus a probiotic blend of Lactobacillus salivarius, Lactobacillus acidophilus and Bifidobacterium bifidus strain BGN4) on ulcerative colitis activity.

Method. Sixty patients with moderate-to-severe ulcerative colitis were enrolled: 30 of them were treated with a single daily oral administration of mesalazine $1200 \mathrm{mg} ; 30$ patients received a single daily oral administration of mesalazine $1200 \mathrm{mg}$ and a double daily administration of a probiotic blend of Lactobacillus salivarius, Lactobacillus acidophilus and Bifidobacterium bifidus strain BGN4. The treatment was carried out for two years and the clinical response evaluated according to the Modified Mayo Disease Activity Index.

Results. All patients treated with combination therapy showed better improvement compared to the controls. In particular, the beneficial effects of probiotics were evident even after two years of treatment.

Conclusions. A long-term treatment modality of anti-inflammatory drugs and probiotics is viable and could be an alternative to corticosteroids in mild-to moderate ulcerative colitis.
\end{abstract}

Key words: bifidobacteria, inflammatory bowel diseases, lactobacilli, microbiota, ulcerative colitis

Received: January 18, 2016; Accepted with revision: August 10, 2016; Available online: September 13, 2016

http://dx.doi.org/10.5507/bp.2016.044

${ }^{a}$ Euro-Mediterranean Institute of Science and Technology (IEMEST), Palermo, Italy

${ }^{b}$ Department of Surgical, Oncological and Stomatological Disciplines, School of Medicine, University of Palermo, Palermo, Italy

'Department of Experimental Biomedicine and Clinical Neuroscience, School of Medicine, University of Palermo, Palermo, Italy

${ }^{d}$ P. Giaccone University Hospital, Palermo, Italy

${ }^{e}$ Department of Anatomy, Cell Biology and Physiology, School of Medidcine, Aamerican University of Beirut, Beirut, Lebanon

Corresponding author: Vincenzo Davide Palumbo, e-mail: vincenzopalumbo@iemest.eu

\section{INTRODUCTION}

Inflammatory bowel disease worldwide incidence and prevalence have been increasing in the last few decades. Ulcerative colitis (UC) is one of the two major types of IBD, along with Crohn disease (CD). Unlike CD, which can affect any part of the gastrointestinal tract, UC characteristically involves the large bowe $1^{1,2}$.

There is a large debate about the exact aetiology of UC. Proposed causes include environmental factors, immune dysfunction, and a likely genetic predisposition. The current hypothesis is that genetically susceptible individuals have abnormalities of humoral and cell-mediated immunity and a generalized enhanced reactivity against commensal intestinal bacteria ${ }^{3}$. This dysregulated mucosal immune response predisposes to colonic inflammation $^{3}$. Whether these abnormalities are the cause or the result of the intense systemic inflammatory response in UC is still unresolved. However, it is well documented that bacterial microflora is altered in patients with active disease $^{4}$. Recent studies reported a great variation in the effects of microbiota, focusing, in particular, on the effects of a pro-inflammatory enterotype on mucosal layer and disease activity ${ }^{5}$.

Modulation of the intestinal microbiota can be performed either by antibiotics or by probiotics, but the former are not good candidates for chronic disease because of antibiotic resistance, potential side effects, and ecological concerns ${ }^{6}$. Therefore, the use of probiotics in IBD could be considered a potential aid to the current conventional therapies. An accurate analysis of scientific data proves that the efficacy of probiotics in the treatment of various diseases has been amply demonstrated and confirmed ${ }^{7-9}$. Actually, several studies have focused on the effects of probiotic blends on enteral microbiota, especially in those cases of dysbiosis, when the normal concentration of "good" bacterial flora is impaired by the presence of pathogenic bacteria ${ }^{10,11}$.

Currently, the standard treatment of UC relies on an initial medical management with corticosteroids and antiinflammatory agents, such as mesalazine, in conjunction with a symptomatic treatment with antidiarrheal agents and rehydration. These treatments have been proven not to be always reliable in controlling the clinical course of 
Table 1. Modified Mayo Disease Activity Index.

\begin{tabular}{|c|c|c|c|c|}
\hline Grade & Bowel frequency & Rectal bleeding & $\begin{array}{l}\text { Physician's global } \\
\text { assessment }\end{array}$ & $\begin{array}{l}\text { Endoscopy/sigmoidoscopy } \\
\text { finding }\end{array}$ \\
\hline 0 & $\begin{array}{l}\text { Normal number of stools } \\
\text { per day for this patient }\end{array}$ & No blood seen & Normal & Normal or inactive disease \\
\hline 1 & $\begin{array}{l}1 \text { or } 2 \text { more stools than } \\
\text { normal }\end{array}$ & $\begin{array}{l}\text { Streaks of blood with stool } \\
\text { less than half the time }\end{array}$ & Mild disease & $\begin{array}{l}\text { Mild disease (erythema, decreased } \\
\text { vascular pattern) }\end{array}$ \\
\hline 2 & $\begin{array}{l}3 \text { or } 4 \text { more stools than } \\
\text { normal }\end{array}$ & $\begin{array}{l}\text { Obvious blood with stool } \\
\text { most of the time }\end{array}$ & Moderate disease & $\begin{array}{l}\text { Moderate disease (marked erythema, } \\
\text { absent vascular pattern, friability, } \\
\text { erosions) }\end{array}$ \\
\hline 3 & $\begin{array}{l}5 \text { or more stools than } \\
\text { normal }\end{array}$ & Blood alone passed & Severe disease & $\begin{array}{l}\text { Severe disease (spontaneous bleeding, } \\
\text { ulceration) }\end{array}$ \\
\hline
\end{tabular}

the disease ${ }^{12,13}$ and present side effects in a significant proportion of patients who do not tolerate the existing treatments ${ }^{14}$. In the present open-labeled randomized controlled study, standard corticosteroid treatment was substituted for a combination therapy (anti-inflammatory + probiotics) for two years, in moderate-to-severe UC and the disease activity was periodically followed-up according to the Modified Mayo Disease Activity Index (MMDAI) (ref. $\left.{ }^{15}\right)$.

\section{MATERIALS AND METHODS}

From January 2011 to December 2012, 60 UC patients were evaluated clinically and endoscopically to establish their disease activity, in accordance with the MMDAI (ref. ${ }^{15}$ ), a simplified composite score incorporating four variables: stool frequency, rectal bleeding, mucosal appearance and physician's rating of disease activity (Table 1). By employing a four point scoring scale for each variable, the relative simplicity of the index reduces the impact of physician and patient subjectivity in disease scoring. Interestingly, the stool frequency score is not an absolute number, but relative to "normal" for that subject. The MMDAI was modified by the deletion of "friability" from an endoscopy score equal to 1; in fact, the assessment of mucosa "friability" is an important subjective parameter and its deletion contributes to the objectivity of the chosen score. Patients over 18 years of age with UC and a moderate-to-severe disease (activity index: 8-12) were considered eligible for the study. The diagnosis of UC, was established on the basis of standard clinical, endoscopic and histological criteria. All subjects were out-patients, attending our Gastroenterology Unit. A condition of steroid dependence, renal impairment, pregnancy, lactation or established low compliance, was considered as an exclusion criterium. The use of other drugs, such as rectal mesalazine or steroid preparations, was not allowed during investigation. Patients were free to leave the study at any time (withdrawal of consent). Other reasons for withdrawal from the investigation were: lack of adherence to the therapeutic schedule or programmed controls $(<85 \%$, poor compliance); onset of symptoms of relapse, confirmed by instrumental procedures (therapeutic failure); onset of drug-related adverse events requiring
Table 2. Modified Mayo Disease Activity Index (MMDAI) from $t_{0}$ to $t_{4}$, in group A, B and A vs B.

\begin{tabular}{crrc}
\hline MMDAI & \multicolumn{1}{c}{ A } & \multicolumn{1}{c}{ B } & A vs B \\
\hline $\mathrm{t}_{0}$ & $10.1 \pm 1.4$ & $10.2 \pm 1.6$ & ns \\
$\mathrm{t}_{1}$ & $7.2 \pm 2.0$ & $6.0 \pm 1.5$ & $P=0.0109$ \\
$\mathrm{t}_{2}$ & $5.4 \pm 1.3$ & $4.8 \pm 0.7$ & $P=0.0232$ \\
$\mathrm{t}_{3}$ & $5.7 \pm 1.4$ & $4.8 \pm 0.8$ & $P=0.0035$ \\
$\mathrm{t}_{4}$ & $6.1 \pm 2.0$ & $4.4 \pm 0.8$ & $P=0.0001$ \\
\hline
\end{tabular}

Table 3. Physician's global assessment from $t_{0}$ to $t_{4}$, in group $\mathrm{A}, \mathrm{B}$ and $\mathrm{A}$ vs $\mathrm{B}$.

\begin{tabular}{cccc}
\hline $\begin{array}{c}\text { Physician's global } \\
\text { assessment }\end{array}$ & $\mathrm{A}$ & $\mathrm{B}$ & $\mathrm{A}$ vs B \\
\hline $\mathrm{t}_{0}$ & $2.6 \pm 0.7$ & $2.7 \pm 0.5$ & $\mathrm{~ns}$ \\
$\mathrm{t}_{1}$ & $1.9 \pm 0.7$ & $1.7 \pm 0.7$ & $\mathrm{~ns}$ \\
$\mathrm{t}_{2}$ & $1.5 \pm 0.6$ & $1.3 \pm 0.5$ & $\mathrm{~ns}$ \\
$\mathrm{t}_{3}$ & $1.4 \pm 0.6$ & $1.2 \pm 0.4$ & $\mathrm{~ns}$ \\
$\mathrm{t}_{4}$ & $1.5 \pm 0.6$ & $1.2 \pm 0.4$ & $P=0.0040$ \\
\hline
\end{tabular}

interruption of treatment. The study was approved by the local ethics committee and all participants subscribed to an informed consent.

The patients were divided into two homogeneous groups: group A, including 22 male and 8 female subjects aged 35-69 years (mean 43 years), was treated pharmacologically with $1200 \mathrm{mg}$ of oral mesalazine once-daily (Mesavancol $^{\circledR} 1200 \mathrm{mg}$ cpr, Giuliani spa, Milan); group B, which included 19 male and 11 female UC patients aged between 28 and 71 years (mean 46 years), was treated with a single daily administration of oral mesalazine 1200 mg (Mesavancol ${ }^{\circledR} 1200$ mg cpr, Giuliani spa, Milan) and a double administration of a probiotic blend of Lactobacillus salivarius, Lactobacillus acidophilus and Bifidobacterium bifidus strain BGN4 (Acronelle ${ }^{\circledR}$, Bromatech srl, Milan, Italy). The treatment was carried out in both groups for two years.

Considering as " $\mathrm{t}_{0}$ " the first evaluation, all patients were subsequently followed-up for $6\left(\mathrm{t}_{1}\right), 12\left(\mathrm{t}_{2}\right), 18\left(\mathrm{t}_{3}\right)$ and $24\left(\mathrm{t}_{4}\right)$ months. A new reassessment of the activity score was carried out at every check-point. Data were evaluated statistically using the ANOVA method for re- 
peated measures (ANOVA Repeated Measures), and the two groups compared by means of $t$ test (unpaired $t$ test).

\section{RESULTS}

During the 24 months of study, patients treated with mesalazine and probiotic blend showed better results than those reached by patients treated with mesalazine alone. In particular, as Table 2 suggests, the benefits of the probiotic blend in combination with the anti-inflammatory treatment are tangible and statistically significant after a period of at least 18 months. Both groups showed an effective improvement of patient general clinical condition which is reflected by a global improvement of their MMDAI, but group B patients advantaged from the use of probiotics which, probably, enhanced the effects of the anti-inflammatory treatment.

Table 3 underlines the positive effects of single daily administration of mesalazine in UC patients. Interestingly, in group B, the anti-inflammatory action of the treatment is evidently powered by probiotics, which ameliorate the clinical response and shorten significantly the time of recovery. Furthermore, the combined therapy contributed to maintain constantly low the score in group B, whose patients showed an acceptable overall clinical condition up to the end of the study. However, a slight deterioration could be observed over time, in group A. Data analysis during the 2 years of treatment in both groups, corroborates the showed results, remarking the slight benefit of the combination therapy compared to the sole use of mesalazine (Table 3).

Stool frequency, the second parameter considered, also showed a significant improvement due to the combined treatment, with a slight reduction of frequency in group B compared to group A (Table 4). Strangely, there was a slight deterioration at 18 months in group $\mathrm{B}$, whereas in group A, even in this case, a moderate loss of effectiveness of the anti-inflammatory therapy could be recognized over time. Comparing the two groups, the reduction of stool frequency became statistically significant in group B, at 6 and 24 months of therapy. The table shows a small reversal of the trend at 18 months, when the patients of group B seemed to lose the advantage supplied by probiotics.

Considering the endoscopic picture (Table 5), both treatments were already effective only after 6 months. In group B, patients showed a significant improvement of intestinal mucosa aspect, compared to group A, and, as already remarked for the overall clinical condition, the beneficial effects of combination therapy remained constant for the entire period of study, compared to group A, whose patients lost some of those benefits already at $t_{3}$.

The evaluation of rectal bleeding more or less followed the same time lapse already seen for the endoscopic picture (Table 6). In fact, bleeding decreased in both groups already at $t_{1}$, but whereas group $B$ enjoyed the positive effects of probiotics up to 2 years, showing a certain constancy of values at each check-point, group A, once again, got progressively worse from 18 months of treatment onwards.
Table 4. Stool frequency from $t_{0}$ to $t_{4}$, in group A, B and $\mathrm{A}$ vs $\mathrm{B}$.

\begin{tabular}{cccc}
\hline $\begin{array}{c}\text { Stool } \\
\text { frequency }\end{array}$ & A & B & A vs B \\
\hline $\mathrm{t}_{0}$ & $2.4 \pm 0.5$ & $2.4 \pm 0.6$ & $\mathrm{~ns}$ \\
$\mathrm{t}_{1}$ & $1.8 \pm 0.6$ & $1.4 \pm 0.5$ & $P=0.0021$ \\
$\mathrm{t}_{2}$ & $1.3 \pm 0.5$ & $1.2 \pm 0.4$ & $\mathrm{~ns}$ \\
$\mathrm{t}_{3}$ & $1.3 \pm 0.5$ & $1.3 \pm 0.5$ & $\mathrm{~ns}$ \\
$\mathrm{t}_{4}$ & $1.5 \pm 0.6$ & $1.0 \pm 0.2$ & $P=0.0006$ \\
\hline
\end{tabular}

Table 5. Endoscopic picture from $t_{0}$ to $t_{4}$, in group A, B and $\mathrm{A}$ vs $\mathrm{B}$.

\begin{tabular}{cccc}
\hline $\begin{array}{c}\text { Endoscopic } \\
\text { finding }\end{array}$ & $\mathrm{A}$ & $\mathrm{B}$ & $\mathrm{A}$ vs B \\
\hline $\mathrm{t}_{0}$ & $2.5 \pm 0.5$ & $2.5 \pm 0.6$ & $\mathrm{~ns}$ \\
$\mathrm{t}_{1}$ & $1.8 \pm 0.8$ & $1.5 \pm 0.6$ & $\mathrm{~ns}$ \\
$\mathrm{t}_{2}$ & $1.3 \pm 0.5$ & $1.2 \pm 0.4$ & $\mathrm{~ns}$ \\
$\mathrm{t}_{3}$ & $1.6 \pm 0.5$ & $1.1 \pm 0.3$ & $P=0.0005$ \\
$\mathrm{t}_{4}$ & $1.6 \pm 0.6$ & $1.1 \pm 0.3$ & $P=0.0005$ \\
\hline
\end{tabular}

Table 6. Rectal bleeding from $t_{0}$ to $t_{4}$, in group A, B and $\mathrm{A}$ vs $\mathrm{B}$.

\begin{tabular}{cccc}
\hline $\begin{array}{c}\text { Rectal } \\
\text { bleeding }\end{array}$ & A & B & A vs B \\
\hline $\mathrm{t}_{0}$ & $2.6 \pm 0.6$ & $2.5 \pm 0.6$ & $\mathrm{~ns}$ \\
$\mathrm{t}_{1}$ & $1.7 \pm 0.7$ & $1.4 \pm 0.5$ & $P=0.0498$ \\
$\mathrm{t}_{2}$ & $1.3 \pm 0.4$ & $1.1 \pm 0.3$ & $\mathrm{~ns}$ \\
$\mathrm{t}_{3}$ & $1.4 \pm 0.5$ & $1.1 \pm 0.3$ & $P=0.0374$ \\
$\mathrm{t}_{4}$ & $1.5 \pm 0.7$ & $1.1 \pm 0.3$ & $P=0.0024$ \\
\hline
\end{tabular}

\section{DISCUSSION}

In the last few years, intestinal microbiota seems to be increasingly involved in UC pathogenesis ${ }^{16,17}$. The characteristic chronic inflammation of the colonic mucosa in $\mathrm{UC}$ is likely due to the constant exposure of the mucosal layer to antigenic endoluminal stimuli. Numerous studies have identified in intestinal dismicrobism the most important endoluminal antigenic stimulus; this, along with the change of the whole intestinal microenvironment, could hyperstimulate the immune system and trigger the inflammatory process ${ }^{18-20}$.

Microbiological studies have detected a significant reduction of Bifidobacteria and Lactobacilli and an overgrowth of specific pathogenic strains, such as Deltaproteobacteria and Bilophila wadsworthia. The use of probiotics in combination with the standard treatment, improves patients' quality of life and life expectancy, reducing significantly clinical symptoms and minimizing side effects ${ }^{21-25}$. The results of our study confirm the beneficial effects of probiotics on UC activity, partly by improving patient's response to anti-inflammatory treatment. Comparing patients treated with probiotic blend and mesalazine, and those patients who received the anti- 
inflammatory alone, data report a significant improvement of MMDAI in the former group, after 18 months. Interestingly, the association of probiotics with mesalazine, seems to guarantee a stable effect in the whole period of observation, different from the anti-inflammatory therapy alone, which seems to be burdened by an unsteady response. Oral 5-ASA administered once daily has been demonstrated to be as effective and safe as conventional dosing (twice or three times daily) for maintenance of remission in quiescent $\mathrm{UC}\left(\mathrm{ref}^{26}\right)$. The choice of testing a specific probiotic blend (Acronelle ${ }^{\circledR}$, Bromatech srl, Milan, Italy) was suggested by the distinguishing features of the bacterial strains contained in the considered probiotic blend: Lactobacillus salivarius has a high anti-inflammatory and antibacterial activity, especially against some of those pathogenic bacterial strains, usually present on the intestinal mucosa of IBD patients ${ }^{27}$; Bifidobacterium bifidum BGN 4 supports Lactobacilli against inflammation, thanks to the chiro-inositol present in its cell membrane, which seems to reduce the production of pro-inflammatory cytokines ${ }^{28}$. Although guidelines ${ }^{29}$ suggest to treat moderate-to-severe UC with corticosteroids, the contemporary use of Mesavancol $\AA$ and Acronelle $\AA$ allowed to reach an encouraging result, avoiding all those therapy side effects which usually decrease patient compliance. For the first time, the long-term efficacy ( 2 years) of such a probiotic blend has been proven, also demonstrating the synergistic effect on disease activity, especially when coupled with anti-inflammatory treatment. Several authors have already contributed to research in this field, however, none of them has followed-up moderate-to-severe UC patients, for a such long period of time. Ishikawa et al. $\left(\right.$ ref. ${ }^{30}$ ) reported that Bifidobacteria-fermented milk (BFM) supplementation reduces the luminal butyrate concentration, a key molecule in the remission of colitis. This reduction reflects the increased uptake or oxidation of SCFAs by the improved colorectal mucosa. Similarly, Kato et al. (ref. ${ }^{31}$ ) found increased levels of faecal butyrate, propionate, and SCFA acid concentrations in patients with active UC (mild to moderate), who received BFM together with conventional treatment. In this pilot study, patients supplemented with BFM showed a significantly lower clinical activity index than the placebo group. Likewise, the post-treatment endoscopic index and histological score were reduced in the BFM group. Probio-Tec AB-25, a mixture of Lactobacillus acidophilus strain La-5 and Bifidobacterium animalis subsp. lactis strain Bb-12, was tested for the maintenance of remission in patients with left-sided UC, in a 1-year, prospective, randomized, double-blind and placebo controlled trial ${ }^{32}$. The safety and tolerance of Probio-Tec AB-25 and the placebo were good. Gastrointestinal symptoms were reported equally in both treatment groups and a relationship between Probio-Tec 25 and gastrointestinal side effects could not be established. At weeks 4 and 28, Bb-12 or La-5 were detected in 11 patients receiving probiotics. Five patients in the probiotic group (25\%) and one patient in the placebo group (8\%) maintained remission after 1 year of treatment. In the probiotic group, the median time to relapse was 125.5 days, versus 104 days in the placebo group. The use of
BIFICO (oral capsules of live enterococci, bifidobacteria, and lactobacilli) in combination with sulphasalazine and glucocorticoid exerts some beneficial effects in preventing the relapse of UC ( ref. $^{33}$ ). The administration of BIFICO plus sulphasalazine and glucocorticoid to UC patients enlarged the number of bifidobacteria and lactobacilli and reduced the number of enterococci, bacteroides, and bifidobacteria present in the faeces compared with the control group. The most studied probiotic in clinical trials is Lactobacillus rhamnosus, which is present in the bowel of healthy individuals. Zocco et al. (ref. ${ }^{34}$ ) studied the efficacy of Lactobacillus rhamnosus GG (LGG) supplementation versus standard mesalazine for maintaining disease remission in UC patients. After 6 and 12 months of treatment, the percentage of patients maintaining clinical remission was, respectively, $91 \%$ and $85 \%$ for the LGG group $(1.8 \times 1010$ viable bacteria/day $), 87 \%$ and $80 \%$ for the mesalazine group ( $2400 \mathrm{mg} / \mathrm{day}$ ), and $94 \%$ and $84 \%$ for the combined treatment (LGG plus mesalazine). The oral administration of Lacteol (Lacteol Fort, Rameda, Egypt), a probiotic preparation that contains $1 \times$ 1010 CFU of Lactobacillus delbrueckii and Lactobacillus fermentum, together with $2400 \mathrm{mg} /$ day of sulfasalazine, during 8 weeks, to UC patients with chronic diarrhea, inhibited the extent of inflammation, prevented mucosal injury, and alleviated colitis ${ }^{35}$. In children with distal active UC, rectal administration of Lactobacillus reuteri ATCC 55730 (as an enema solution containing $1 \times 1010$ CFU) for 8 weeks in addition to standard oral mesalazine, resulted in a significant decrease in the MDAI compared with the children that received the corresponding placebo. In addition, all of the children on Lactobacillus reuteri had a clinical response, whereas only $53 \%$ of the children on the placebo responded. Clinical remission was achieved in $31 \%$ of the Lactobacillus reuteri group and in no children of the placebo group ${ }^{36}$. D'Incà et al. (ref. ${ }^{37}$ ) evaluated the effect of an 8-week oral and/or rectal administration of Lactobacillus casei DG on colonic-associated microbiota, mucosal cytokine balance, and TLR expression in patients with mild left-sided UC. The patients were divided into three groups: the first group received oral 5-ASA alone, the second group received oral 5-ASA plus oral Lactobacillus casei DG $(8 \times 108 \mathrm{CFU})$, and the third group received oral 5-ASA and rectal Lactobacillus casei DG $(8 \times 108 \mathrm{CFU})$. A significant improvement of the histological scores was found in patients receiving the probiotic strain by the oral or rectal route of administration. Nevertheless, oral supplementation with Lactobacillus casei DG did not have a significant effect on the counts of Enterobacteriaceae or Lactobacillus. However, the occurrence of Lactobacillus and Enterobacteriaceae cultured from biopsy specimens was increased and decreased, respectively, in the group that took the probiotic rectally. Moreover, the rectal administration of Lactobacillus casei DG significantly reduced TLR-4 and IL- $1 \beta$ levels and significantly increased mucosal IL-10.

Probiotics act into the inflamed intestine, destroying "bad" bacterial flora and restoring previous micro-environment conditions. Probably, such an effect this prevents that abnormal reaction of the human immune system at 
the base of intestinal autoimmune diseases. In this case, patients treated with probiotics showed an overall improvement of all studied parameters: patients showed a better clinical response, reduced significantly their stool frequency, maintained easily their haemoglobin values and exhibited a significant improvement of their gut mucosa condition. Likely, a random choice of probiotic strains should not be the right way to cure IBD definitely, but the present study demonstrates the efficacy of some "good" bacterial strains in assisting anti-inflammatory drug mechanism of action.

Author contributions: VDP: study design, results analysis; MR, AILM, GT: study design, final approval; AMG, FC, PD: results analysis; GD, SB: literature search.

Conflict of interest statement: None declared.

\section{REFERENCES}

1. Molodecky NA, Soon IS, Rabi DM, Ghali WA, Ferris M, Chernoff G, Benchimol El, Panaccione R, Ghosh S, Barkema HW, Kaplan GG. Increasing incidence and prevalence of the inflammatory bowel diseases with time, based on systematic review. Gastroenterology 2012;142:46-54.

2. Cotran RS, Collins T, Robbins SL, Kumar V. Pathologic Basis of Disease, 3rd edn. Philadelphia: WB Saunders; 1998.

3. Xavier RJ, Podolsky DK. Unravelling the pathogenesis of inflammatory bowel disease. Nature 2007;448:427-34.

4. Almeida MG, Kiss DR, Zilberstein B, Quintanilha AG, Teixeira MG, Habr-Gama A. Intestinal mucosa-associated microflora in ulcerative colitis patients before and after restorative proctocolectomy with an ileoanal pouch. Dis Colon Rectum 2008;51:1113-9.

5. Hildebrand F, Nguyen TL, Brinkman B, Yunta RG, Cauwe B Vandenabeele $P$, Liston $A$, Raes J. Inflammation-associated enterotypes, host genotype cage and inter-individual effects drive gut microbiota variation in common laboratory mice. Genome Biol 2013;14:R4.

6. Tursi A, Brandimarte G, Papa A, Giglio A, Elisei W, Giorgetti GM, Forti G, Morini S, Hassan C, Pistoia MA, Modeo ME, Rodino' S, D'Amico T, Sebkova L, Sacca' N, Di Giulio E, Luzza F, Imeneo M, Larussa T, Di Rosa S, Annese V, Danese S, Gasbarrini A. Treatment of relapsing mild-tomoderate ulcerative colitis with the probiotic VSL\#3 as adjunctive to a standard pharmaceutical treatment: a double blind, randomized, placebo-controlled study. Am J Gastroenterol 2010;105:2218-27.

7. Tomasello G, Palumbo VD, Miceli A, Sinagra E, Bruno A, Abruzzo A, Cappello F, Patti AM, Giglio RM, Damiani P, Tomasello R, Noto M, Arculeo VM, Accardo MF, Lo Monte Al. Probiotics and conventiona therapy: new frontiers in therapeutic approach in articular manifestations of IBD. Progr Nutr 2014;16:176-87.

8. Sinagra E, Tomasello G, Cappello F, Leone A, Cottone M, Bellavia M, Rossi F, Facella T, Damiani P, Zeenny MN, Damiani F, Abruzzo A Damiano G, Palumbo VD, Cocchi M, Jurjus A, Spinelli G, Lo Monte Al, Raimondo D. Probiotics, prebiotics and synbiotics in inflammatory bowel diseases: state-of-the-art and new insights. J Biol Regul Homeost Agents 2013;27:919-33.

9. Kvasnovsky CL, Bjarnason I, Papagrigoriadis S. What colorectal surgeons should know about probiotics: a review. Colorectal Dis 2015; 17:840-8.

10. Mack DR. Probiotics in inflammatory bowel diseases and associated conditions. Nutrients 2011;3:245-64.

11. D'Haens GR, Sartor RB, Silverberg MS, Petersson J, Rutgeerts P. Future directions in inflammatory bowel disease management. J Crohns Colitis 2014;8:726-34.

12. Langan RC, Gotsch PB, Krafczyk MA, Skillinge DD. Ulcerative colitis: diagnosis and treatment. Am Fam Physician 2007;76:1323-30.

13. MacDermott RP, Green JA, Ashley CC. What is the optimal therapy for severe ulcerative colitis? Inflamm Bowel Dis 2008;14:S228-31.

14. Miele E, Pascarella F, Giannetti E, Quaglietta L, Baldassano RN, Staiano A. Effect of a probiotic preparation (VSL\#3) on induction and maintenance of remission in children with ulcerative colitis. Am J Gastroenterol 2009;104:437-43.

15. Scherl EJ, Pruitt R, Gordon GL, Lamet M, Shaw A, Huang S, Mareya S, Forbes WP. Safety and efficacy of a new $3.3 \mathrm{~g}$ b.i.d. tablet formulation in patients with mild-to-moderately-active ulcerative colitis: a multicenter, randomized, double-blind, placebo-controlled study. Am J Gastroenterol 2009;104:1452-9.

16. Bellavia M, Damiano G, Gioviale MC, Palumbo VD, Cacciabaudo F Buscemi G, Lo Monte Al. Abnormal expansion of segmented filamentous bacteria in the gut: a role in pathogenesis of chronic inflammatory intestinal diseases? Rev Microbiol 2011;22:45-7.

17. Tomasello G, Bellavia M, Palumbo VD, Gioviale MC, Damiani P, Lo Monte Al . From gut microflora imbalance to mycobacteria infection: is there a relationship with chronic intestinal inflammatory diseases? Ann Ital Chir 2011;82:361-8.

18. Rodolico V, Tomasello G, Zerilli M, Martorana A, Pitruzzella A, Gammazza AM, David S, Zummo G, Damiani P, Accomando S, Conway de Macario E, Macario AJ, Cappello F. Hsp60 and Hsp10 increase in colon mucosa of Crohn's disease and ulcerative colitis. Cell Stress Chaperones 2010;15:877-84.

19. Tomasello G, Rodolico V, Zerilli M, Martorana A, Bucchieri F, Pitruzzella A, Marino Gammazza A, David S, Rappa F, Zummo G, Damiani P, Accomando S, Rizzo M, de Macario EC, Macario AJ, Cappello F. Changes in immunohistochemical levels and subcellular localization after therapy and correlation and colocalization with CD68 suggest a pathogenetic role of $\mathrm{Hsp60}$ in ulcerative colitis. Appl Immunohistochem Mol Morphol 2011;19:552-61.

20. Tomasello G, Sciumé C, Rappa F, Rodolico V, Zerilli M, Martorana A, Cicero G, De Luca R, Damiani P, Accardo FM, Romeo M, Farina F, Bonaventura G, Modica G, Zummo G, Conway de Macario E, Macario AJ, Cappello F. Hsp10, Hsp70, and Hsp90 immunohistochemical levels change in ulcerative colitis after therapy. Eur J Histochem 2011;55:e38.

21. Yoshimatsu Y, Yamada A, Furukawa R, Sono K, Osamura A, Nakamura K, Aoki H, Tsuda Y, Hosoe N, Takada N, Suzuki Y.Effectiveness of probiotic therapy for the prevention of relapse in patients with inactive ulcerative colitis. World J Gastroenterol 2015;21:5985-94.

22. Chen WX, Ren LH, Shi RH. Enteric micobiota leads to new therapeutic strategies for ulcerative colitis. World J Gastroenterol 2014;20:1565763.

23. Saez-Lara MJ, Gomez-Llorente C, Plaza-Diaz J, Gil A.The role of probiotic lactic acid bacteria and bifidobacteria in the prevention and treatment of inflammatory bowel disease and other related diseases: a systematic review of randomized human clinical trials. Biomed Res Int 2015;2015:505878.

24. Strisciuglio C, Miele E, Giugliano FP, Vitale S, Andreozzi M, Vitale A, Catania MR, Staiano A, Troncone R, Gianfrani C. Probiotic bacteria enhance antigen sampling and processing by dendritic cells in pediatric Inflammatory Bowel Disease. Dig Liver Dis 2015;21:1491-8.

25. Tralongo $P$, Tomasello G, Sinagra E, Damiani $P$, Leone A, Palumbo VD, Giammanco M, Di Majo D, Damiani F, Abruzzo A, Bruno A, Cassata G, Cicero L, Noto M, Tomasello R, Lo Monte Al. The role of butyric acid as a protective agent against inflammatory bowel diseases. EMBJ 2014;9:24-35.

26. Sandborn WJ, Korzenik J, Lashner B, Leighton JA, Mahadevan U, Marion JF, Safdi M, Sninsky CA, Patel RM, Friedenberg KA, Dunnmon P, Ramsey D, Kane S. Once-daily dosing of delayed-release oral mesalamine (400-mg tablet) is as effective as twice-daily dosing for maintenance of remission of ulcerative colitis. Gastroenterology 2010;138:1286-96.

27. Collins JK. Demonstration of functional properties of probiotic lactic acid bacteria. Ind Latte 2001;37:39-61.

28. You HJ, Oh DK, Ji GE. Anticancerogenic effect of a novel chiroinositol-containing polysaccharide from Bifidobacterium bifidum BGN4. FEMS Microbiol Lett 2004;240:131-6.

29. Bressler B, Marshall JK, Bernstein CN, Bitton A, Jones J, Leontiadis Gl, Panaccione R, Steinhart AH, Tse F, Feagan B, Toronto Ulcerative Colitis Consensus Group. Clinical practice guidelines for the medical management of nonhospitalized ulcerative colitis: the Toronto consensus. Gastroenterology 2015;148:1035-58.

30. Ishikawa H, Akedo I, Umesaki Y, Tanaka R, Imaoka A, Otani T. Randomized controlled trial of the effect of bifidobacteria-fermented milk on ulcerative colitis. J Am Coll Nutr 2003;22:56-63.

31. Kato K, Mizuno S, Umesaki Y, Ishii Y, Sugitani M, Imaoka A, Otsuka M, Hasunuma O, Kurihara R, Iwasaki A, Arakawa Y. Randomized 
placebocontrolled trial assessing the effect of bifidobacteria-fermented milk on active ulcerative colitis. Aliment Pharmacol Ther 2004;20:1133-41.

32. Wildt S, Nordgaard I, Hansen U, Brockmann E, Rumessen JJ. A randomised double-blind placebo-controlled trial with Lactobacillus acidophilus La-5 and Bifidobacterium animalis subsp. lactis BB-12 for maintenance of remission in ulcerative colitis. J Crohns Colitis 2011;5:115-21.

33. Cui HH, Chen CL, Wang JD, Yang YJ, Cun Y, Wu JB, Liu YH, Dan HL, Jian $Y T$, Chen XQ. Effects of probiotic on intestinal mucosa of patients with ulcerative colitis. World J Gastroenterol 2004;10:1521-5.

34. Zocco MA, dal Verme LZ, Cremonini F, Piscaglia AC, Nista EC, Candelli M, Novi M, Rigante D, Cazzato IA, Ojetti V, Armuzzi A, Gasbarrini G,
Gasbarrini A. Efficacy of Lactobacillus GG in maintaining remission of ulcerative colitis. Aliment Pharmacol Ther 2006:23:1567-74.

35. Hegazy SK, El-Bedewy MM. Effect of probiotics on pro-inflammatory cytokines and NF-kB activation in ulcerative colitis. World J Gastroenterol 2010;16:4145-51.

36. Oliva S, Di Nardo G, Ferrari F, Mallardo S, Rossi P, Patrizi G, Cucchiara S, Stronati L. Randomised clinical trial: the effectiveness of Lactobacillus reuteri ATCC 55730 rectal enema in children with active distal ulcerative colitis. Aliment Pharmacol Ther 2012;35:327-34.

37. D'Incà $R$, Barollo $M$, Scarpa $M$, Grillo $A R$, Brun $P$, Vettorato $M G$, Castagliuolo I, Sturniolo GC. Rectal administration of Lactobacillus casei DG modifies flora composition and toll-like receptor expression in colonic mucosa of patients with mild ulcerative colitis. Dig Dis Sci 2011;56:1178-87. 